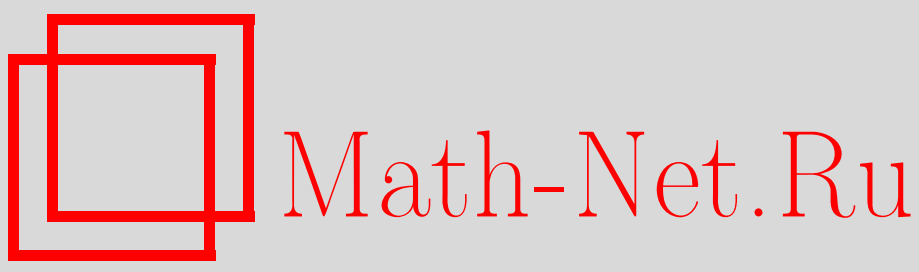

В. И. Буслаев, О теореме Ван Флека для правильных $C$-дробей с предельно периодическими коэффициентами, Изв. РАН. Сер. матем., 2001, том 65, выпуск 4, 35-48

DOI: https://doi.org/10.4213/im346

Использование Общероссийского математического портала Math-Net.Ru подразумевает, что вы прочитали и согласны с пользовательским соглашением

http://www . mathnet.ru/rus/agreement

Параметры загрузки:

IP: 18.207 .199 .55

26 апреля 2023 г., $17: 27: 32$ 
УДК 517.524

\author{
В.И. Буслаев
}

\title{
О теореме Ван Флека для правильных $C$-дробей с предельно периодическими коэффициентами
}

\begin{abstract}
В статье исследуется множество сходимости правильной $C$-дроби с предельно периодическими коэффициентами. Указанное исследование проводится на основе общего утверждения о сходимости композиций дробно-линейных преобразований, коэффициенты которых являются голоморфными по параметру предельно периодическими функциями. Показано, что множество особенностей указанных $C$-дробей обладает экстремальным свойством, формулируемым в терминах трансфинитного диаметра (емкости) множеств.

Библиографйя: 7 наименований.
\end{abstract}

\section{§1. Формулировка результата}

В теории непрерывных дробей хорошо известна следующая теорема Ван Флека [1] о сходимости правильной $C$-дроби с предельно постоянными коэффициентами, т.е. функциональной непрерывной дроби вида

$$
\frac{a_{1} z}{1+\frac{a_{2} z}{1+\cdots}}
$$

где $a_{1}, a_{2}, \ldots$ - комплексные числа, имеющие предел.

ТЕОРемА (Ван Флек). Пусть коэффициенты $a_{1}, a_{2}, \ldots$ правильной $C$-дроби (1) имеют предел $\lim _{n \rightarrow \infty} a_{n}=a \neq 0$. Тогда $C$-дробъ (1) сходится $\kappa$ функиии $f(z)$ в области $D=\mathbb{C} \backslash \Gamma$, где $\Gamma=\left\{z \in \mathbb{C}: z=-\frac{1}{4 a} t, t \geqslant 1\right\}, \quad f(z)$ мероморфна в $D$ и сходимость равномерна на любом компакте, лежащем в $D$ и не содержащем полюсов $f(z)$.

Весьма важное замечание к теореме Ван Флека о невозможности мероморфного продолжения предельной функции $f(z)$ ни через какой интервал луча Г было сделано А. А. Гончаром.

ЗАмечАние (А.А. Гончар). В предположениях и обозначениях теоремы Ван $\Phi$ лека и дополнительном предположении $a_{n} \neq 0, n=1,2, \ldots$, предельная функция $f(z)$ не может иметь мероморфного продолжения ни в какую область $(\mathbb{C} \backslash \Gamma) \cup$ $\left\{\left|z-z_{0}\right|<\varepsilon\right\}$, где $z_{0} \in \Gamma, \varepsilon-$ произвольное сколь угодно малое положительное число.

Работа выполнена при финансовой поддержке ИНТАС (проект № 2000-272), РФФИ (грант № 99-01-01149) и Российской программы поддержки ведущих научных школ (грант № 00-15-96132).

(C) В.И. БуслАев, 2001 
В данной статье будет вьписано в явном виде множество сходимости правильной $C$-дроби с предельно периодическими коэффициентами (т.е. с коэффициентами $a_{1}, a_{2}, \ldots$, имеюшими периодические пределы $\lim _{n \rightarrow \infty} a_{n m+l}=a^{l}$, где $m$ - некоторое фиксированное натуральное число, $l=1, \ldots, m)$ и будут доказаны утверждения, аналогичные приведенным вьше в теореме Ван Флека и в замечании Гончара. Этот результат будет получен как следствие более общего утверждения о сходимости композиций (по $w) S_{1} \circ \cdots \circ S_{n}(w, z)$ дробно-линейных (по $\left.w\right)$ преобразований

$$
S_{n}(w, z)=\frac{a_{n}(z) w+b_{n}(z)}{c_{n}(z) w+d_{n}(z)}
$$

с голоморфными предельно периодическими коэффициентами $a_{n}(z), b_{n}(z), c_{n}(z)$, $d_{n}(z)$.

Пусть коэффициенты $a_{n}(z), b_{n}(z), c_{n}(z), d_{n}(z)$ дробно-линейных (по $\left.w\right)$ преобразований (2) являются голоморфными по $z$ функциями в некоторой области $\Omega$ комплексной плоскости и имеют равномерные на компактах, лежащих в $\Omega$, периодические пределы

$$
\begin{aligned}
& \lim _{n \rightarrow \infty} a_{n m+l}(z)=a^{l}(z), \quad \lim _{n \rightarrow \infty} b_{n m+l}(z)=b^{l}(z), \\
& \lim _{n \rightarrow \infty} c_{n m+l}(z)=c^{l}(z), \quad \lim _{n \rightarrow \infty} d_{n m+l}(z)=d^{l}(z),
\end{aligned}
$$

где $m$ - некоторое фиксированное натуральное число, $l=1, \ldots, m$. Будем пользоваться далее следуюшими обозначениями:

$$
\begin{gathered}
\left(\begin{array}{cc}
\alpha^{l}(z) & \beta^{l}(z) \\
\gamma^{l}(z) & \delta^{l}(z)
\end{array}\right)=\left(\begin{array}{cc}
a^{l}(z) & b^{l}(z) \\
c^{l}(z) & d^{l}(z)
\end{array}\right) \times \cdots \times\left(\begin{array}{cc}
a^{m}(z) & b^{m}(z) \\
c^{m}(z) & d^{m}(z)
\end{array}\right) \\
\times\left(\begin{array}{cc}
a^{1}(z) & b^{1}(z) \\
c^{1}(z) & d^{1}(z)
\end{array}\right) \times \cdots \times\left(\begin{array}{cc}
a^{l-1}(z) & b^{l-1}(z) \\
c^{l-1}(z) & d^{l-1}(z)
\end{array}\right), \\
I(z)=\alpha^{l}(z)+\delta^{l}(z), \quad \Delta(z)=\alpha^{l}(z) \delta^{l}(z)-\beta^{l}(z) \gamma^{l}(z), \\
\Gamma=\left\{z \in \Omega: I^{2}(z)=4 t \Delta(z), 0 \leqslant t \leqslant 1\right\} .
\end{gathered}
$$

Легко видеть, что функции $I(z)$ и $\Delta(z)$ - это соответственно следи определитель матрицы $\left(\begin{array}{cc}\alpha^{l}(z) & \beta^{l}(z) \\ \gamma^{l}(z) & \delta^{l}(z)\end{array}\right)$, равной произведению матрищ $\left(\begin{array}{cc}a^{j}(z) & b^{j}(z) \\ c^{j}(z) & d^{j}(z)\end{array}\right)$, взятых в круговом порядке, $j=l, \ldots, m, 1, \ldots, l-1$. Поэтому $I(z)$ и $\Delta(z)$ не зависят от $l$. При этом, очевидно,

$$
\Delta(z)=\prod_{j=1}^{m}\left(a^{j}(z) d^{j}(z)-b^{j}(z) c^{j}(z)\right) .
$$

Легко видеть также, что множество Г можно определить как множество, состоящее из тех $z \in \Omega$, при которых либо $I(z)=0$, либо $1-4 \Delta(z) I^{-2}(z)$ принимает отрицательные (или нулевые) значения, и как множество

$$
\left\{z \in \Omega:\left|I(z)+\sqrt{I^{2}(z)-4 \Delta(z)}\right|=\left|I(z)-\sqrt{I^{2}(z)-4 \Delta(z)}\right|\right\} .
$$


Предполагая, что

$$
\left|I(z)+\sqrt{I^{2}(z)-4 \Delta(z)}\right|<\left|I(z)-\sqrt{I^{2}(z)-4 \Delta(z)}\right|
$$

при $z \in \Omega \backslash \Gamma$, обозначим

$$
\begin{gathered}
p^{l}(z)=\frac{\alpha^{l}(z)-\delta^{l}(z)+\sqrt{I^{2}(z)-4 \Delta(z)}}{2 \gamma^{l}(z)}, \quad z \in \Omega \backslash \Gamma, \\
\Lambda=\bigcap_{l=1}^{m}\left\{(w, z) \in \overline{\mathbb{C}} \times(\Omega \backslash \Gamma): w \neq p^{l}(z)\right\} .
\end{gathered}
$$

Отметим, что введенные обозначения допускают следующую интерпретацию. Введем в рассмотрение дробно-линейные преобразования

$$
S^{l}(w, z)=\frac{a^{l}(z) w+b^{l}(z)}{c^{l}(z) w+d^{l}(z)}, \quad l=1, \ldots, m,
$$

и их круговые композиции (по $w$ )

$$
\widetilde{S}^{l}(w, z)=S^{l} \circ \cdots \circ S^{m} \circ S^{1} \circ \cdots \circ S^{l-1}(w, z) .
$$

Тогда

$$
\widetilde{S}^{l}(w, z)=\frac{\alpha^{l}(z) w+\beta^{l}(z)}{\gamma^{l}(z) w+\delta^{l}(z)}, \quad l=1, \ldots, m,
$$

$\Gamma$ - множество тех $z \in \Omega$, при которых неподвижные точки

$$
p_{1,2}^{l}(z)=\frac{\alpha^{l}(z)-\delta^{l}(z) \pm \sqrt{I^{2}(z)-4 \Delta(z)}}{2 \gamma^{l}(z)}
$$

преобразований $\widetilde{S}^{l}(w, z)$ нейтральны, а $p^{l}(z)$ при $z \in \Omega \backslash \Gamma$ и указанном выборе знака корня - отталкивающая неподвижная точка $\widetilde{S}^{l}(w, z)$.

В статье будет доказана следующая

ТЕОРема 1. Пусть имеются дробно-линейнье (nо w) преобразования (2) такие, что их коэффичиенты $a_{n}(z), b_{n}(z), c_{n}(z), d_{n}(z)$ являются голомор $\phi-$ ныцми по z функциями в некоторой области $\Omega$ комплексной плоскости и существуют равномерные на компактах, лежащих в $\Omega$, периодические предельb $\lim _{n \rightarrow \infty} a_{n m+l}(z)=a^{l}(z), \lim _{n \rightarrow \infty} b_{n m+l}(z)=b^{l}(z), \quad \lim _{n \rightarrow \infty} c_{n m+l}(z)=$ $c^{l}(z), \lim _{n \rightarrow \infty} d_{n m+l}(z)=d^{l}(z)$, где $m$ - некоторое фиксированное натуральное число, $l=1, \ldots, m$. Тогда во введенных выце обозначениях имеют место следующие утверждения:

1) при всех $(w, z) \in \Lambda$ последовательность композиций (nо $w) S_{1} \circ \ldots$ $\cdots \circ S_{n}(w, z)$ сходится при $n \rightarrow \infty \kappa$ функции $f(z)$;

2) предельная функиия $f(z)$ не зависит от $w$ и является мероморфной по $z$ функиией в $\Omega \backslash \Gamma$; 
3) сходимость равномерна в сферической метрике на компактах, лежаu, ux 8 в $\Lambda$.

Фиксируем произвольное число $w_{0} \in \overline{\mathbb{C}}$. Из теоремы 1 следует, в частности, что при $n \rightarrow \infty$ последовательность композиций $S_{1} \circ \cdots \circ S_{n}\left(w_{0}, z\right)$ сходится к функции $f(z)$ при всех $z$ таких, что $\left(w_{0}, z\right) \in \Lambda$, т.е. при всех $z \in \Omega \backslash\left(\Gamma \cup E\left(w_{0}\right)\right)$, где $E\left(w_{0}\right)=\bigcup_{l=1}^{m}\left\{z \in \Omega: w_{0}=p^{l}(z)\right\}$, и при этом предельная мероморфная функция $f(z)$ может быть мероморфно продолжена в $\Omega \backslash \Gamma$. Воспользуемся далее этим замечанием при $w_{0}=0$. Хорошо известно, что последовательность подходящих дробей непрерьвной дроби (1) совпадает с последовательностью композищий $S_{1} \circ \cdots \circ S_{n}(0, z)$ дробно-линейных преобразований $S_{n}(w, z)=\frac{a_{n} z}{w+1}$. В предположениях и обозначениях теоремы 1 для такого вида дробно-линейных преобразований имеем

$$
\Delta(z)=(-1)^{m} a^{1} \ldots a^{m} z^{m}
$$

где $a^{l}=\lim _{n \rightarrow \infty} a_{n m+l}, \quad l=1, \ldots, m$. Поэтому фактически как частный случай теоремы 1 будут получены все, кроме последнего, утверждения в следующей теореме о сходимости правильной $C$-дроби с предельно периодическими коэффициентами. В этой теореме для следа (суммы диагональных элементов) матрицы $A$ используется обозначение $\operatorname{Tr} A$, для целой части действительного числа $\alpha$ используется обозначение $[\alpha]$, а под $[a, b]$ понимается отрезок с концами в точках $a$ и $b$.

ТЕОРемА 2. Пусть коэффициенты правильной $C$-дроби (1) имеют периодические предель $\lim _{n \rightarrow \infty} a_{n m+l}=a^{l}, l=1, \ldots, m$, где $m$ - некоторое фиксированное натуральное число. Тогда:

1) $C$-дробь (1) сходится $к$ функиии $f(z)$ на множестве $D=\mathbb{C} \backslash(\Gamma \cup E)$, где

$$
\begin{gathered}
\Gamma=\Gamma\left(a^{1}, \ldots, a^{m}\right)=\left\{z \in \mathbb{C}: z^{-m} I^{2}\left(z ; a^{1}, \ldots, a^{m}\right) \in\left[0,4(-1)^{m} a^{1} \ldots a^{m}\right]\right\} \\
I\left(z ; a^{1}, \ldots, a^{m}\right)=\operatorname{Tr}\left(\left(\begin{array}{cc}
0 & a^{1} z \\
1 & 1
\end{array}\right) \times \cdots \times\left(\begin{array}{cc}
0 & a^{m} z \\
1 & 1
\end{array}\right)\right)
\end{gathered}
$$

- алгебраический многочлен степени не выше $[\mathrm{m} / 2]$ с единичным.м свободным членом, $E=E\left(a^{1}, \ldots, a^{m}\right)$ - конечное множество (в обозначениях теоремы $\left.1, E=\bigcup_{l=1}^{m}\left\{z \in \mathbb{C}: 0=p^{l}(z)\right\}\right)$, состоящее из не более чем $m[(m-1) / 2]$ точек, в частности $E$ - пустое множсество при $m=1,2$;

2) мнохсество сходимости $D$ является связной областью и содержит точку $z=0$;

3) $f(z)$ мероморфна в $D$ и сходимость равномерна на компактах, лежащих в $D$ и не содержаших полюсов $f(z)$;

4) $f(z)$ допускает мероморфное продолжсение в $\mathbb{C} \backslash \Gamma$;

5) если Г - ограниченное множество, т.е. если

$$
\lim _{z \rightarrow \infty} z^{-m} I^{2}\left(z ; a^{1}, \ldots, a^{m}\right) \notin\left[0,4(-1)^{m} a^{1} \ldots a^{m}\right]
$$

то т четно и $f(z)$ допускает мероморфное продолжение в точку $z=\infty$;

6) пусть имеют место дополнительные предположсения $a_{n} \neq 0, \quad n=$ $1,2, \ldots$, и пусть $F$ - компакт комплексной плоскости такой, что росток 
функции $f\left(z^{-1}\right)$ в точке $z=\infty$ (очевидно, существующий, так как $\left.0 \in D\right)$ допускает мероморфное продолэсение в компоненту $\mathbb{C} \backslash F$, содержащую точку $z=\infty ;$ тогда $d(F) \geqslant d\left(\Gamma^{-1}\right)=\left|a^{1} \ldots a^{m}\right|^{1 / m}$, где $d(F)$ и $d\left(\Gamma^{-1}\right)-$ трансфинитные диаметры компактов $F u \Gamma^{-1}=\left\{z: z^{-1} \in \Gamma\right\}$ соответственно.

Определение трансфинитного диаметра (емкости) множеств комплексной плоскости и его основные свойства см., например, в [2, с. 285].

В силу утверждения 4) теоремы 2 все точки конечного множества $E=$ $E\left(a^{1}, \ldots, a^{m}\right)$ являются устранимыми особыми точками предельной мероморфной функции $f(z)$, и по этой причине множество $E$ не очень интересно. Значительно более интересным является множество $\Gamma=\Gamma\left(a^{1}, \ldots, a^{m}\right)$. Легко видеть, что при $m=1 \Gamma(a)$ - это луч, определенньй в теореме Ван Флека, а при $m=2$

$$
\begin{aligned}
\Gamma\left(a^{1}, a^{2}\right) & =\left\{z \in \mathbb{C}: z^{-2}\left(1+a^{1} z+a^{2} z\right)^{2} \in\left[0,4 a^{1} a^{2}\right]\right\} \\
& =\left\{z \in \mathbb{C}: z=-\left(a^{1}+2 t \sqrt{a^{1} a^{2}}+a^{2}\right)^{-1},-1 \leqslant t \leqslant 1\right\}
\end{aligned}
$$

- это дуга окружности (или прямой), соединяющая точки $z_{1,2}=-\left(\sqrt{a^{1}} \pm \sqrt{a^{2}}\right)^{-2}$, причем продолжение этой дуги проходит через точку $z=0$. Легко видеть также, что если какой-либо из пределов $a^{l}, l=1, \ldots, m$, равен нулю, то $\Gamma=\{z \in \mathbb{C}$ : $\left.z^{-m} I\left(z ; a^{1}, \ldots, a^{m}\right)^{2}=0\right\}$ - конечное множество, состоящее из не более чем $[m / 2]$ точек.

Очевидно, что без дополнительных предположений $a_{n} \neq 0, n=1,2, \ldots$, означающих, что все дробно-линейные преобразования $S_{n}(w, z)=\frac{a_{n} z}{w+1}, z \neq 0$, невырождены, утверждение 6) может и не выполняться, так как, например, при $a_{1}=0$ предельная функция $f(z)$ тождественно равна нулю. Отметим два следствия утверждения 6) об экстремальности трансфинитного диаметра компакта $\Gamma^{-1}$. Первое из них распространяет замечание Гончара к теореме Ван Флека на случай $C$-дробей с предельно периодическими коэффициентами.

СлЕДСТВИЕ 1. В предположсениях и обозначениях теоремы 2 и дополнительных предположениях $a_{n} \neq 0, \quad n=1,2, \ldots, \quad и a^{1} \ldots a^{m} \neq 0$ функиия $f(z)$ не может иметь мероморфного продолжения ни в какую область $(\mathbb{C} \backslash \Gamma) \cup\left\{\left|z-z_{0}\right|<\varepsilon\right\}$, где $z_{0} \in \Gamma, \quad \varepsilon>0$.

Действительно, предположим противное. Тогда функция $f\left(z^{-1}\right)$ допускает мероморфное продолжение в некоторую область $\left(\mathbb{C} \backslash \Gamma^{-1}\right) \cup\left\{\left|z-z_{0}^{-1}\right|<\delta\right\}$, где $z_{0}^{-1} \in \Gamma^{-1}, \delta>0$. Так как при $a^{1} \ldots a^{m} \neq 0$ имеем строгое неравенство $d\left(\Gamma^{-1} \backslash\left\{\left|z-z_{0}^{-1}\right|<\delta\right\}\right)<d\left(\Gamma^{-1}\right)$, то это противоречит утверждению 6$)$.

СлЕДСТВИЕ 2. Пусть $P(z)$ - алгебраический многочлен, $k$ равно 0 или 1 , $K=\left\{z \in \mathbb{C}: z^{k} P^{2}(z) \in[0,1]\right\}$ и $\Phi$ - множество всех компактов $F$ комплексной плоскости таких, что функиия $\sqrt{P^{2}(z)-z^{-k}}$ допускает вьделение регулярной ветви в $\mathbb{C} \backslash F$. Тогда $K \in \Phi$ и $d(K)=\min _{F \in \Phi} d(F)$.

Действительно, пусть $P(z)=A z^{q}+\cdots$, где $A \neq 0$. Не теряя обшности рассуждений, можно считать, что $q \geqslant 1$, так как иначе следствие 2 тривиально. Положим $m=2 q+k$ и подберем $m$ параметров $a^{1}, \ldots, a^{m}$ таким образом, чтобы $z^{q} I\left(z^{-1} ; a^{1}, \ldots, a^{m}\right)=A^{-1} P(z)$ и $4(-1)^{m} a^{1} \ldots a^{m}=A^{-2}$ (напомним, что по 
утверждению 1$)$ теоремы $2 I\left(z^{-1} ; a^{1}, \ldots, a^{m}\right)$ - многочлен от $z^{-1}$ степени не выше $[m / 2]=q$ с единичным свободным членом, и, следовательно, для $m$ свободных параметров $a^{1}, \ldots, a^{m}$ имеем $q+1 \leqslant m$ условий). Тогда

$$
\begin{aligned}
\Gamma^{-1}\left(a^{1}, \ldots, a^{m}\right) & =\left\{z: z^{m} I^{2}\left(z^{-1} ; a^{1}, \ldots, a^{m}\right) \in\left[0,4(-1)^{m} a^{1} \ldots a^{m}\right]\right\} \\
& =\left\{z: z^{k} A^{-2} P^{2}(z) \in\left[0, A^{-2}\right]\right\}=K .
\end{aligned}
$$

Рассмотрим далее периодическую $C$-дробь (1) такую, что $a_{n m+l}=a^{l}$ при всех $n=1,2, \ldots, l=1, \ldots, m$. Для такой периодической $C$-дроби предельная функция $f(z)$ легко находится в явном виде как притягивающая неподвижная точка дробно-линейного преобразования

$$
\widetilde{S}^{1}(w, z)=S^{1} \circ \cdots \circ S^{m}(w, z),
$$

где $S^{l}(w, z)=\frac{a^{l} z}{w+1}, \quad l=1, \ldots, m$. В обозначениях теоремы 1 точки ветвления функции $f\left(z^{-1}\right)$ совпадают с точками ветвления функции

$$
\sqrt{I^{2}\left(z^{-1}\right)-4 \Delta\left(z^{-1}\right)}=\sqrt{A^{-2} P^{2}(z) z^{-2 q}-A^{-2} z^{-m}}=A^{-1} z^{-q} \sqrt{P^{2}(z)-z^{-k}} .
$$

Поэтому из утверждения 6) теоремы 2 получаем следствие 2.

Заметим, что в рассмотренном выше чисто периодическом случае теорема 2 совпадает с известной теоремой Шталя [3] о сходимости диагональных аппроксимаций Паде алгебраических функций вне разрезов комплексной плоскости минимального трансфинитного диаметра, превращающих алгебраическую функцию в регулярную. Но все-таки подчеркнем, что теорема 2 и теорема Шталя имеют разные области применимости. Теорема 2 применима не только в чисто периодическом случае, но и в предельно периодическом случае, а теорема Шталя применима не только к алгебраическим функциям рассмотренного выше вида, но и к произвольным алгебраическим функциям. Отметим также, что в отличие от теоремы Шталя в теореме 2 выписывается и явный вид разрезов минимального трансфиниитного диаметра. Для иллюстрации следствия 2 рассмотрим алгебраическую функцию с четырьмя точками ветвления второго порядка. Легко видеть, что следствие 2 применимо, если эти четыре точки ветвления лежат в вершинах $\left((z+a)^{2}+b\right)^{2}+c=0$ некоторого параллелограмма (случай $k=0$ ), либо если одна из точек ветвления равна нулю, а три другие находятся как решения кубического уравнения вида $z(z+a)^{2}+c=0$ (случай $\left.k=1\right)$. В первом случае множество

$$
K=\left\{z \in \mathbb{C}:\left((z+a)^{2}+b\right)^{2}+c t=0,0 \leqslant t \leqslant 1\right\}
$$

состоит из двух непересекающихся кусков гиперболы, каждый из которых соединяет две ближайшие друг к другу соседние вершины параллелограмма. Если параллелограмм является ромбом, то эти два куска гиперболы вырождаются в диагонали ромба. Во втором случае $(k=1)$ имеем

$$
K=\left\{z \in \mathbb{C}: z(z+a)^{2}+c t=0,0 \leqslant t \leqslant 1\right\} .
$$

В качестве примера второго случая выберем следуюшие четыре точки ветвления: $z_{1}=0, z_{2}=2, z_{3,4}= \pm i$. В этом случае множество

$$
K=\left\{z \in \mathbb{C}: z(z-1)^{2}-2 t=0,0 \leqslant t \leqslant 1\right\}
$$

состоит из отрезка $[0,2]$ и кривой, проходящей через точки $\pm i$ и $1 / 3$. 


\section{§2. Доказательство теорем 1 и 2}

Основу доказательства теоремы 1 будет составлять следующее утверждение, которое выделим в самостоятельную лемму.

ЛЕмма 1. Пусть дробно-линейные преобразования $S_{n}(w)=\frac{\alpha_{n} w+\beta_{n}}{\gamma_{n} w+\delta_{n}}$ таковы, что при некоторых $p \in \mathbb{C}, 0<q<1 u \theta>0$ выполняются неравенства

$\left|\delta_{n}+p \gamma_{n}\right|+\theta^{-1}\left|\gamma_{n} p^{2}+\left(\delta_{n}-\alpha_{n}\right) p-\beta_{n}\right| \leqslant q\left(\left|\alpha_{n}-p \gamma_{n}\right|-\theta\left|\gamma_{n}\right|\right), \quad n=1,2, \ldots$

Тогда при всех $w$ таких, что $|w-p|>\theta$, существует предел $\lim _{n \rightarrow \infty} S_{1} \circ \ldots$ $\cdots \circ S_{n}(w)=S$. При әтом $S$ не зависит от $w,|S-p| \geqslant \theta u$

$$
\left|\left(S_{1} \circ \cdots \circ S_{n}(w)-p\right)^{-1}-(S-p)^{-1}\right| \leqslant q^{n} \frac{|w-p|+\theta}{|w-p|-\theta} \theta^{-1} .
$$

Приведем также и другую, по сушеству эквивалентную, формулировку леммы 1.

ЛЕмма 2. Пусть дробно-линейные преобразования $S_{n}(w)=\frac{\alpha_{n} w+\beta_{n}}{\gamma_{n} w+\delta_{n}}$ таковы, что при некоторых $p \in \mathbb{C}, 0<q<1$ и $\theta>0$ выполняются неравенства

$\left|\alpha_{n}-p \gamma_{n}\right|+\theta\left|\gamma_{n}\right| \leqslant q\left(\left|\delta_{n}+p \gamma_{n}\right|-\theta^{-1}\left|\gamma_{n} p^{2}+\left(\delta_{n}-\alpha_{n}\right) p-\beta_{n}\right|\right), \quad n=1,2, \ldots$

Тогда при всех $w$ таких, что $|w-p|<\theta$, существует предел $\lim _{n \rightarrow \infty} S_{1} \circ \ldots$ $\cdots \circ S_{n}(w)=S$. При әтом $S$ не зависит от $w,|S-p| \leqslant \theta u$

$$
\left|S_{1} \circ \cdots \circ S_{n}(w)-S\right| \leqslant q^{n} \frac{\theta+|w-p|}{\theta-|w-p|} \theta .
$$

Частный случай $p=0, \theta=1$ леммы 2 для невырожденных дробно-линейных преобразований $S_{n}(w)$ доказан автором в [4, с. 17]. Если же среди дробно-линейных преобразований $S_{n}(w)$ имеются вырожденные, то указанный частный случай тривиален. Покажем теперь, что и лемма 1 и лемма 2 представляют собой по сути дела разные формулировки этого частного случая $(p=0, \theta=1)$ леммы 2 , получаемые простой заменой переменной.

ДоКАЗАТЕЛЬСТво ЛЕмм 1 и 2 . Положим $T_{n}(w)=\mathscr{Q}^{-1} \circ S_{n} \circ \mathscr{Q}(w)$, где $\mathscr{Q}(w)=\theta w^{-1}+p$ в случае леммы 1 и $\mathscr{Q}(w)=\theta w+p$ в случае леммы 2 . В первом случае

$$
T_{n}(w)=\frac{\left(\delta_{n}+\gamma_{n} p\right) w+\theta \gamma_{n}}{\theta^{-1}\left(-\gamma_{n} p^{2}+\left(\alpha_{n}-\delta_{n}\right) p+\beta_{n}\right) w+\left(\alpha_{n}-\gamma_{n} p\right)},
$$

а во втором случае

$$
T_{n}(w)=\frac{\left(\alpha_{n}-\gamma_{n} p\right) w+\theta^{-1}\left(-\gamma_{n} p^{2}+\left(\alpha_{n}-\delta_{n}\right) p+\beta_{n}\right)}{\theta \gamma_{n} w+\left(\delta_{n}+\gamma_{n} p\right)} .
$$

В любом случае из предположений лемм следует, что коэффициенты дробно-линейных преобразований $T_{n}(w)=\frac{a_{n} w+b_{n}}{c_{n} w+d_{n}}$ удовлетворяют условию $\left|a_{n}\right|+\left|c_{n}\right| \leqslant$ $q\left(\left|d_{n}\right|-\left|b_{n}\right|\right)$ уже доказанного в [4] частного случая $p=0, \theta=1$ леммы 2 . Поэтому 
при всех $|w|<1$ существует не зависяший от $w$ предел $\lim _{n \rightarrow \infty} T_{1} \circ \cdots \circ T_{n}(w)=T$. При этом $|T| \leqslant 1$ и

$$
\left|T_{1} \circ \cdots \circ T_{n}(w)-T\right| \leqslant q^{n} \frac{1+|w|}{1-|w|}, \quad|w|<1, \quad n=1,2, \ldots
$$

Так как $S_{1} \circ \cdots \circ S_{n}(w)=\mathscr{Q} \circ T_{1} \circ \cdots \circ T_{n} \circ \mathscr{Q}^{-1}(w)$, то отсюда следует, что при всех $w$ таких, что $\left|\mathscr{Q}^{-1}(w)\right|<1$, существует не зависящий от $w$ предел $\lim _{n \rightarrow \infty} S_{1} \circ \cdots \circ S_{n}(w)=S=\mathscr{Q}(T)$. При этом

$$
\left|\mathscr{Q}^{-1}(S)\right|=|T| \leqslant 1
$$

и

$$
\begin{aligned}
\left|\mathscr{Q}^{-1} \circ S_{1} \circ \cdots \circ S_{n}(w)-\mathscr{Q}^{-1}(S)\right| & =\left|T_{1} \circ \cdots \circ T_{n} \circ \mathscr{Q}^{-1}(w)-T\right| \\
& \leqslant q^{n} \frac{1+\left|\mathscr{Q}^{-1}(w)\right|}{1-\left|\mathscr{Q}^{-1}(w)\right|}, \quad n=1,2, \ldots
\end{aligned}
$$

Вспоминая определение дробно-линейного преобразования $\mathscr{Q}(w)$, отсюда получаем утверждения лемм.

ДОКАЗАТЕЛЬСТВо ТЕОРЕМЫ 1. Заметим, что $p^{l}(z)=S^{l} \circ \cdots \circ S^{m}\left(p^{1}(z), z\right)$ и равенство $\widetilde{S}^{l}(w, z)=p^{l}(z)$ выполняется тогда и только тогда, когда $w=p^{l}(z)$, $l=1, \ldots, m$. Поэтому

$$
\Lambda=\bigcap_{l=1}^{m}\left\{(w, z) \in \overline{\mathbb{C}} \times(\Omega \backslash \Gamma): S^{1} \circ \cdots \circ S^{l}(w, z) \neq p^{1}(z)\right\} .
$$

Легко видеть, что теорема 1 будет доказана, если для любой точки $z_{0} \in \Omega \backslash$ Г будет найдено положительное число $r=r\left(z_{0}\right)$ такое, что композиции $S_{1} \circ \ldots$ $\cdots \circ S_{n}(w, z)$ равномерно в сферической метрике сходятся при $n \rightarrow \infty$ на компактах вида

$$
\Lambda\left(z_{0}, r, \tau\right)=\bigcap_{l=1}^{m}\left\{(w, z) \in \overline{\mathbb{C}} \times K\left(z_{0}, r\right):\left|S^{1} \circ \cdots \circ S^{l}(w, z)-p^{1}(z)\right| \geqslant \tau\right\}
$$

где $K\left(z_{0}, r\right)=\left\{\left|z-z_{0}\right| \leqslant r\right\}, \tau$-произвольное сколь угодно малое положительное число, к не зависящей от $w$ и мероморфной по $z$ в круге $K\left(z_{0}, r\right)$ функции $f(z)$.

Фиксируем точку $z_{0} \in \Omega \backslash \Gamma$. Переходя в случае необходимости от заданных дробно-линейных преобразований $(2)$ к дробно-линейным преобразованиям $S_{n, t}(w, z)=\mathscr{Q}_{t}^{-1} \circ S_{n} \circ \mathscr{Q}_{t}(w, z)$, где $\mathscr{Q}_{t}(w)=w^{-1}+t$ с надлежаще выбранным числом $t$, можно считать, не теряя общности рассуждений, что $\gamma^{1}\left(z_{0}\right) \neq 0$.

Выберем в качестве $r\left(z_{0}\right)$ любое положительное число такое, что круг $K=$ $K\left(z_{0}, r\right)$ принадлежит $\Omega \backslash \Gamma$ и $\gamma^{1}(z) \neq 0$ при всех $z \in K$. Для комплексного числа $z$, не лежащего на отрицательной полуоси, обозначим через $\sqrt{z}$ тот из корней, для которого число $1+\sqrt{z}$ имеет меньший модуль, т.е. $|1+\sqrt{z}|<|1-\sqrt{z}|$. Тогда $\sqrt{z}-$ 
функция, регулярная во всей комплексной плоскости с разрезом по отрищательной полуоси. Легко видеть, что

$$
p^{1}(z)=\frac{\alpha^{1}(z)-\delta^{1}(z)+I(z) \sqrt{1-4 \Delta(z) I^{-2}(z)}}{2 \gamma^{1}(z)}
$$

- функция, голоморфная в некоторой окрестности круга $K$, и при всех $z \in K$ выполняется неравенство

$$
\begin{aligned}
& 2^{-1}\left|\delta^{1}(z)+\gamma^{1}(z) p^{1}(z)\right|=\left|I(z)\left(1+\sqrt{1-4 \Delta(z) I^{-2}(z)}\right)\right|< \\
& \quad<\left|I(z)\left(1-\sqrt{1-4 \Delta(z) I^{-2}(z)}\right)\right|=2^{-1}\left|\alpha^{1}(z)-\gamma^{1}(z) p^{1}(z)\right| .
\end{aligned}
$$

Из (5) следует, в частности, что голоморфная функция $\alpha^{1}(z)-\gamma^{1}(z) p^{1}(z)$ не принимает при $z \in K$ значений, равных нулю, и, следовательно,

$$
\min _{z \in K}\left|\alpha^{1}(z)-\gamma^{1}(z) p^{1}(z)\right|>0 .
$$

Фиксируем $\tau_{0}>0$ и компакт $\Lambda_{0}=\Lambda\left(z_{0}, r\left(z_{0}\right), \tau_{0}\right)$. Так как компакт $\Lambda_{0}$ лежит в $\Lambda$ и так как коэффициенты дробно-линейных преобразований $S_{n m+1} \circ \cdots \circ S_{n m+l}(w, z)$ равномерно сходятся на $K$ при $n \rightarrow \infty$ к коэффициентам $S^{1} \circ \cdots \circ S^{l}(w, z)$, то из (4) получаем, что найдется индекс $N_{1}$ такой, что при всех $n \geqslant N_{1}$, всех $l=1, \ldots, m$ и всех $(w, z) \in \Lambda_{0}$

$$
\left|S_{n m+1} \circ \cdots \circ S_{n m+l}(w, z)-p^{1}(z)\right| \geqslant v,
$$

где $v>0$.

Из неравенств (5) и (6) следует, что найдутся положительные числа $\theta<v$ и $s<1$ такие, что при всех $z \in K$

$$
\left|\delta^{1}(z)+\gamma^{1}(z) p^{1}(z)\right|+\theta\left|\gamma^{1}(z)\right| \leqslant s\left|\alpha^{1}(z)-\gamma^{1}(z) p^{1}(z)\right| .
$$

Положим

$$
\left(\begin{array}{cc}
\alpha_{n}^{1}(z) & \beta_{n}^{1}(z) \\
\gamma_{n}^{1}(z) & \delta_{n}^{1}(z)
\end{array}\right)=\left(\begin{array}{ll}
a_{n m+1}(z) & b_{n m+1}(z) \\
c_{n m+1}(z) & d_{n m+1}(z)
\end{array}\right) \times \cdots \times\left(\begin{array}{ll}
a_{n m+m}(z) & b_{n m+m}(z) \\
c_{n m+m}(z) & d_{n m+m}(z)
\end{array}\right) .
$$

По условиям теоремы голоморфные в $\Omega \backslash \Gamma$ функщии $\alpha_{n}^{1}(z), \beta_{n}^{1}(z), \gamma_{n}^{1}(z), \delta_{n}^{1}(z)$ и $\gamma_{n}^{1}(z)\left(p^{1}(z)\right)^{2}+\left(\delta_{n}^{1}(z)-\alpha_{n}^{1}(z)\right) p^{1}(z)-\beta_{n}^{1}$ равномерно сходятся на $K$ соответственно к функциям $\alpha^{1}(z), \beta^{1}(z), \gamma^{1}(z), \delta^{1}(z)$ и $\gamma^{1}(z)\left(p^{1}(z)\right)^{2}+\left(\delta^{1}(z)-\alpha^{1}(z)\right) p^{1}(z)-$ $\beta^{1}(z) \equiv 0$. Поэтому из (6) и (8) следует, что найдутся положительное число $q<1$ и индекс $N \geqslant N_{1}$ такие, что при всех $n \geqslant N$ и всех $z \in K$

$$
\begin{aligned}
& \left|\delta_{n}^{1}(z)+\gamma_{n}^{1}(z) p^{1}(z)\right|+\theta^{-1}\left|\gamma_{n}^{1}(z)\left(p^{1}(z)\right)^{2}+\left(\delta_{n}^{1}(z)-\alpha_{n}^{1}(z)\right) p^{1}(z)-\beta_{n}^{1}(z)\right| \\
& \quad+\theta\left|\gamma_{n}^{1}(z)\right| \leqslant q\left|\alpha_{n}^{1}(z)-\gamma_{n}^{1}(z) p^{1}(z)\right| .
\end{aligned}
$$

Это означает, что для последовательности дробно-линейных (по $w$ ) преобразований

$$
\begin{gathered}
\widetilde{S}_{n}^{1}(w, z)=S_{n m+1} \circ \cdots \circ S_{n m+m}(w, z)=\frac{\alpha_{n}^{1}(z) w+\beta_{n}^{1}(z)}{\gamma_{n}^{1}(z) w+\delta_{n}^{1}(z)} \\
z \in K, \quad n=N, N+1, \ldots,
\end{gathered}
$$


и чисел $p=p^{1}(z), q$ и $\theta$ выполнены условия леммы 1 . Поэтому по лемме 1 при всех $(w, z) \in \tilde{\Lambda}=\left\{(w, z) \in \overline{\mathbb{C}} \times K:\left|w-p^{1}(z)\right| \geqslant v\right\}$ (напомним, что $v>\theta$ ) существует не зависяший от $w$ предел

$$
\lim _{n \rightarrow \infty} \widetilde{S}_{N}^{1} \circ \cdots \circ \widetilde{S}_{n}^{1}(w, z)=f_{N}(z)
$$

композиций дробно-линейных преобразований $\widetilde{S}_{n}^{1}(w, z), n=N, N+1, \ldots$, причем

$$
\left|f_{N}(z)-p^{1}(z)\right|^{-1} \leqslant \theta^{-1}
$$

И

$$
\begin{aligned}
& \left|\left(\widetilde{S}_{N}^{1} \circ \cdots \circ \widetilde{S}_{n}^{1}(w, z)-p^{1}(z)\right)^{-1}-\left(f_{N}(z)-p^{1}(z)\right)^{-1}\right| \\
& \quad \leqslant q^{n-N+1} \frac{\left|w-p^{1}(z)\right|+\theta}{\left|w-p^{1}(z)\right|-\theta} \theta^{-1} \leqslant q^{n-N+1} \frac{v+\theta}{v-\theta} \theta^{-1} .
\end{aligned}
$$

Пусть $w_{0} \in \overline{\mathbb{C}}$ таково, что $\left|w_{0}\right|>v+\max _{z \in K}\left|p^{1}(z)\right|$. Тогда $\left(w_{0}, z\right) \in \tilde{\Lambda}$ при всех $z \in K$. Из неравенств (9) и (10) имеем, в частности, ограниченность в круге $K$ мероморфных по $z$ функций $\left(\widetilde{S}_{N}^{1} \circ \cdots \circ \widetilde{S}_{n}^{1}\left(w_{0}, z\right)-p^{1}(z)\right)^{-1}, n=N, N+1, \ldots$, а следовательно, и их голоморфность в круге $K$. Из неравенства (10) следует, что и функция $\left(f_{N}(z)-p^{1}(z)\right)^{-1}$ голоморфна в круге $K$ как равномерный предел голоморфных функций $\left(\widetilde{S}_{N}^{1} \circ \cdots \circ \widetilde{S}_{n}^{1}\left(w_{0}, z\right)-p^{1}(z)\right)^{-1}$. Таким образом, $f_{N}(z)$ мероморфна в круге $K$.

Пусть $n=k m+l, k \geqslant N, 1 \leqslant l \leqslant m$. Тогда в силу (7) $\left(S_{k m+1} \circ \cdots\right.$ $\left.\cdots \circ S_{k m+l}(w, z), z\right) \in \tilde{\Lambda}$ при всех $(w, z) \in \Lambda_{0}$. Поэтому из неравенства (10) получаем, что композиции

$$
S_{N m+1} \circ \cdots \circ S_{n}(w, z)=\widetilde{S}_{N}^{1} \circ \cdots \circ \widetilde{S}_{k-1}^{1}\left(S_{k m+1} \circ \cdots \circ S_{k m+l}(w, z), z\right)
$$

равномерно в сферической метрике сходятся при $n \rightarrow \infty$ к мероморфной функции $f_{N}(z)$ на компакте $\Lambda_{0}$. Отсюда стандартным образом получаем существование равномерного на $\Lambda_{0}$ в сферической метрике предела

$$
\begin{aligned}
\lim _{n \rightarrow \infty} S_{1} \circ \cdots \circ S_{n}(w, z) & =S_{1} \circ \cdots \circ S_{N m}\left(\lim _{n \rightarrow \infty} S_{N m+1} \circ \cdots \circ S_{n}(w, z), z\right) \\
& =S_{1} \circ \cdots \circ S_{N m}\left(f_{N}(z), z\right)=f(z),
\end{aligned}
$$

где предельная функция $f(z)=S_{1} \circ \cdots \circ S_{N m}\left(f_{N}(z), z\right)$, очевидно, мероморфна в круге $K$. Теорема 1 доказана.

ДоКАЗАТЕЛЬСТво ТЕОРЕМЫ 2. Как уже отмечалось, сходимость непрерывной $C$-дроби (1) эквивалентна сходимости последовательности композиций $S_{1} \circ \cdots \circ S_{n}(0, z)$ дробно-линейных (по $w$ ) преобразований $S_{n}(w, z)=\frac{a_{n} z}{w+1}$. Положим в теореме $1 \Omega=\mathbb{C}, S_{n}(w, z)=\frac{a_{n} z}{w+1}$ и заметим, что в обозначениях теорем 1 и 2

$$
\begin{gathered}
\Delta(z)=(-1)^{m} a^{1} \ldots a^{m} z^{m}, \quad I(z)=I\left(z ; a^{1}, \ldots, a^{m}\right), \\
\Gamma=\left\{z \in \Omega: I^{2}(z)=4 t \Delta(z), 0 \leqslant t \leqslant 1\right\} \\
=\left\{z \in \mathbb{C}: I^{2}\left(z ; a^{1}, \ldots, a^{m}\right)=4 t(-1)^{m} a^{1} \ldots a^{m} z^{m}, 0 \leqslant t \leqslant 1\right\} \\
=\left\{z \in \mathbb{C}: z^{-m} I^{2}\left(z ; a^{1}, \ldots, a^{m}\right) \in\left[0,4(-1)^{m} a^{1} \ldots a^{m}\right]\right\}=\Gamma\left(a^{1}, \ldots, a^{m}\right) .
\end{gathered}
$$


По теореме $1 C$-дробь (1) сходится на множестве $D=\mathbb{C} \backslash(\Gamma \cup E)$, где $E=$ $\bigcup_{l=1}^{m}\left\{z \in \mathbb{C}: 0=p^{l}(z)\right\}$ к функции $f(z)$, мероморфной в $\mathbb{C} \backslash \Gamma$, равномерно на компактах, лежаших в $D$ и не содержащих полюсов $f(z)$.

Покажем далее, что $I\left(z ; a^{1}, \ldots, a^{m}\right)$ является многочленом степени не вьше $[m / 2]$ с единичным свободным членом. Индукцией по $m$ легко показывается, что

$$
\begin{aligned}
& \left(\begin{array}{cc}
0 & a^{1} z \\
1 & 1
\end{array}\right) \times \cdots \times\left(\begin{array}{cc}
0 & a^{m} z \\
1 & 1
\end{array}\right) \\
& \quad=\left(\begin{array}{cc}
a^{1} z t_{m-3}\left(z ; a^{3}, \ldots, a^{m-1}\right) & a^{1} z t_{m-2}\left(z ; a^{3}, \ldots, a^{m}\right) \\
t_{m-2}\left(z ; a^{2}, \ldots, a^{m-1}\right) & t_{m-1}\left(z ; a^{2}, \ldots, a^{m}\right)
\end{array}\right),
\end{aligned}
$$

где $t_{-2}=0, t_{-1}=t_{0}=1$ и

$$
\begin{aligned}
& t_{m}\left(z ; a^{1}, \ldots, a^{m}\right)=1+z \sum_{l=1}^{m} a^{l}+z^{2} \sum_{l_{0}<l_{1}-1} a^{l_{0}} a^{l_{1}} \\
& +z^{3} \sum_{\substack{l_{0}<l_{1}-1<l_{2}-2 \\
l^{[(m+1) / 2]}}} a^{l_{0}} a^{l_{1}} a^{l_{2}}+\ldots \\
& \quad \sum_{l_{0}<l_{1}-1<\cdots<l_{[(m-1) / 2]}-[(m-1) / 2]} a^{l_{0}} \ldots a^{l_{[(m-1) / 2]},}, m=1,2, \ldots
\end{aligned}
$$

Непосредственно из определения видно, что $t_{m}\left(z ; a^{1}, \ldots, a^{m}\right)$ - алгебраический многочлен степени не выше $[(m+1) / 2]$ с единичным свободным членом. Следовательно, $I\left(z ; a^{1}, \ldots a^{m}\right)=a^{1} z t_{m-3}\left(z ; a^{3}, \ldots, a^{m-1}\right)+t_{m-1}\left(z ; a^{2}, \ldots, a^{m}\right)$ - алгебраический многочлен степени не выше $[\mathrm{m} / 2]$ с единичным свободным членом.

Покажем теперь, что множество $E=E\left(a^{1}, \ldots, a^{m}\right)=\bigcup_{l=1}^{m}\left\{0=p^{l}(z)\right\}$ состоит из не более чем $m[(m-1) / 2]$ точек и не содержит точку $z=0$. Учитьвая (11), имеем

$$
\begin{aligned}
\widetilde{S}^{1}(w, z) & =S^{1} \circ \ldots \circ S^{m}(w, z) \\
& =\frac{a^{1} z t_{m-3}\left(z ; a^{3}, \ldots, a^{m-1}\right) w+a^{1} z t_{m-2}\left(z ; a^{3}, \ldots, a^{m}\right)}{t_{m-2}\left(z ; a^{2}, \ldots, a^{m-1}\right) w+t_{m-1}\left(z ; a^{2}, \ldots, a^{m}\right)} .
\end{aligned}
$$

Так как $p^{1}(z)$ - неподвижная точка дробно-линейного преобразования $\widetilde{S}^{1}(w, z)$, то из (12) получаем

$$
\left\{p^{1}(z)=0\right\} \subset\left\{\widetilde{S}^{1}(0, z)=0\right\}=\left\{a^{1} z t_{m-2}\left(z ; a^{3}, \ldots, a^{m}\right)=0\right\} .
$$

Легко видеть также, что $0 \notin\left\{p^{1}(z)=0\right\}$, так как $w=0$ является притягивающей, а не отталкивающей точкой дробно-линейного преобразования $\widetilde{S}^{1}(w, 0)=\frac{0 w+0}{w+1}$. Поэтому $\left\{p^{1}(z)=0\right\} \subset\left\{t_{m-2}\left(z ; a^{3}, \ldots, a^{m}\right)\right\}$. Так как $t_{m-2}\left(z ; a^{3}, \ldots, a^{m}\right)-$ многочлен степени не выше $[(m-1) / 2]$, то множество $\left\{p^{1}(z)=0\right\}$ состоит из не более чем $[(m-1) / 2]$ точек. Аналогичньм образом множество $\left\{p^{l}(z)=0\right\}$ не содержит точку $z=0$ и состоит из не более чем $[(m-1) / 2]$ точек. Следовательно, множество $E=\bigcup_{l=1}^{m}\left\{0=p^{l}(z)\right\}$ не содержит точку $z=0$ и состоит из не более чем $m[(m-1) / 2]$ точек и, в частности, пусто при $m=1,2$. 
Так как $0 \notin E$, а непосредственно из определения $\Gamma=\Gamma\left(a^{1}, \ldots, a^{m}\right)$ следует, что и $0 \notin \Gamma$, то $0 \in D$ (впрочем, утверждение $0 \in D$, очевидно, следует и из известного критерия Ворпицкого [5] о сходимости непрерывной дроби (1) при $|z|<$ $\left.\left(4 \varlimsup_{n \rightarrow \infty}\left|a_{n}\right|\right)^{-1}\right)$. Таким образом, утверждения 1$\left.), 3\right)$ и 4), а также включение $0 \in D$ доказаны.

Докажем теперь утверждение 5) в предположении (3). При нечетных $m$ имеем $\operatorname{deg} I^{2}\left(z ; a^{1}, \ldots, a^{m}\right) \leqslant 2[m / 2]<m$ и, следовательно,

$$
\lim _{z \rightarrow \infty} z^{-m} I^{2}\left(z ; a^{1}, \ldots, a^{m}\right)=0 \in\left[0,4(-1)^{m} a^{1} \ldots a^{m}\right],
$$

т.е. (3) невозможно при нечетных $m$. Таким образом, $m$ четно. Применим теперь теорему 1 к последовательности

$$
\breve{S}_{n}(w, z)=\frac{\alpha_{n}^{1}(z) z^{-m / 2} w+\beta_{n}^{1}(z) z^{-m / 2}}{\gamma_{n}^{1}(z) z^{-m / 2} w+\delta_{n}^{1}(z) z^{-m / 2}}
$$

где

$$
\left(\begin{array}{cc}
\alpha_{n}^{1}(z) & \beta_{n}^{1}(z) \\
\gamma_{n}^{1}(z) & \delta_{n}^{1}(z)
\end{array}\right)=\left(\begin{array}{cc}
0 & a_{n m+1} z \\
1 & 1
\end{array}\right) \times \cdots \times\left(\begin{array}{cc}
0 & a_{n m+m} z \\
1 & 1
\end{array}\right) .
$$

Заметим, что по аналогии с равенством (12) многочлены $\alpha_{n}^{1}(z), \beta_{n}^{1}(z), \gamma_{n}^{1}(z), \delta_{n}^{1}(z)$ являются многочленами степени не вьше $m / 2$ (при четных $m$ ). Поэтому коэффициенты дробно-линейных преобразований $\breve{S}_{n}(w, z)$ являются голоморфными в $\overline{\mathbb{C}} \backslash\{0\}$ функциями. Так как $\breve{I}(z)=z^{-m / 2} I\left(z ; a^{1}, \ldots, a^{m}\right)$ и $\breve{\Delta}(z)=(-1)^{m} a^{1} \ldots a^{m}$, то из (3) следует, что $\breve{I}^{2}(z) \neq 4 t \breve{\Delta}(z), 0 \leqslant t \leqslant 1$, при всех $|z|>R$, включая $z=\infty$, где $R$ - некоторое достаточно большое число. По теореме 1 имеем, что при любом фиксированном $w$ последовательность композиций $\breve{S}_{1}^{1} \circ \cdots \circ \breve{S}_{n}^{1}(w, z)$ сходится на множестве $\{|z|>R\} \backslash \breve{E}(w)$, где $\breve{E}(w)$ - некоторое зависящее от $w$ конечное множество точек, к мероморфной функции $\breve{f}(z)$, совпадающей, очевидно, с $f(z)$ и допускающей мероморфное продолжение на все множество $\{|z|>R\}$, включая точку $z=\infty$.

Докажем теперь утверждение 6) при помоши двух хорошо известных результатов. Первый из них - это формулы, выражаюшие коэффициенты $a_{n}$ правильной $C$-дроби (1) (при условии $a_{n} \neq 0, n=1,2, \ldots$ ) через ганкелевы определители предельной функции $f(z)$ (см., например, [6, с. 220, 221]). Если $f(z)=\sum_{n=1}^{\infty} f_{n} z^{n}$ в некоторой окрестности точки $z=0$, то из этих формул следует, в частности, что $H_{n}=\prod_{k=0}^{n-1}\left(a_{1} \ldots a_{2 k+1}\right)$, где

$$
H_{n}=\left|\begin{array}{ccc}
f_{1} & \ldots & f_{n} \\
\ldots \ldots & \ldots & \ldots \\
f_{n} & \ldots & f_{2 n-1}
\end{array}\right| .
$$

Поэтому из условия существования периодических пределов коэффициентов $a_{n}$ имеем

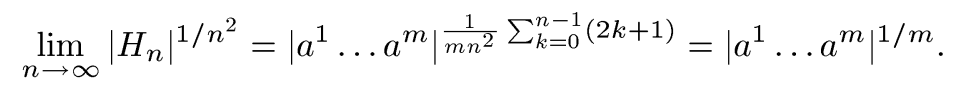

Второй результат, лежаший в основе доказательства утверждения 6), - это известная теорема Полиа [7] об оценке верхнего предела ганкелевых определителей голоморфной функции посредством трансфинитного диаметра множества ее особенностей. 
ТЕОРема (Полиа). Пусть $G$ - компакт комплексной плоскости, и пусть $g(z)$ - функция, голоморфная в компоненте $\mathbb{C} \backslash G$, содержащей точку $z=\infty$. Если $g(z)=\sum_{n=1}^{\infty} g_{n} z^{-n}$ при достаточно больших z, то

$$
\varlimsup_{n \rightarrow \infty}\left|\begin{array}{ccc}
g_{1} & \ldots & g_{n} \\
\ldots \ldots & \ldots & \ldots \\
g_{n} & \ldots & g_{2 n-1}
\end{array}\right|^{1 / n^{2}} \leqslant d(G),
$$

где $d(G)$ - трансфинитный диаметр компакта $G$.

Легко видеть, что теорема Полиа сформулирована для голоморфных функций, но верна и для мероморфных в $\mathbb{C} \backslash G$ функций, так как добавление к $G$ конечного числа точек не меняет его трансфинитного диаметра, а точками накопления полюсов могут служить только лишь точки компакта $G$. Пусть компакт $F$ такой, как указано в утверждении 6). Тогда, применяя теорему Полиа к функции $g(z)=f\left(z^{-1}\right)=\sum_{n=1}^{\infty} f_{n} z^{-n}$, имеем

$$
\varlimsup_{n \rightarrow \infty}\left|H_{n}\right|^{1 / n^{2}} \leqslant d(F) .
$$

Отсюда и из равенства (13) получаем $d(F) \geqslant\left|a^{1} \ldots a^{m}\right|^{1 / m}$.

Покажем теперь, что $d\left(\Gamma^{-1}\right)=\left|a^{1} \ldots a^{m}\right|^{1 / m}$. Это равенство следует непосредственно из определения множества Г и хорошо известных свойств трансфинитного диаметра. Действительно, известно, что трансфинитный диаметр любого отрезка равен четверти его длины и что для всякого компакта $E$ и всякого алгебраического многочлена $p_{m}(z)$ степени $m$ с единичным старшим коэффициентом справедливо равенство $d\left(\left\{z: p_{m}(z) \in E\right\}\right)=d(E)^{1 / m}$ (см., например, [2, с. 290]). Так как $z^{m} I^{2}\left(a^{1} z^{-1}, \ldots, a^{m} z^{-1}\right)$ - алгебраический многочлен степени $m$ с единичным старшим коэффициентом, то

$$
\begin{aligned}
d\left(\Gamma^{-1}\right) & =d\left(\left\{z: z^{m} I^{2}\left(z^{-1} ; a^{1}, \ldots, a^{m}\right) \in\left[0,4(-1)^{m} a^{1} \ldots a^{m}\right]\right\}\right) \\
& =d\left(\left[0,4(-1)^{m} a^{1} \ldots a^{m}\right]\right)^{1 / m}=\left|a^{1} \ldots a^{m}\right|^{1 / m} .
\end{aligned}
$$

Утверждение 6) доказано.

Завершим теперь доказательство утверждения 2), а именно докажем, что множество $D=\mathbb{C} \backslash(\Gamma \cup E)$ сходимости $C$-дроби (1) связно. Так как множество $E$ конечно, то для этого достаточно показать, что связно множество $\mathbb{C} \backslash \Gamma$. Так как $\Gamma=\Gamma\left(a^{1}, \ldots, a^{m}\right)$ зависит только лишь от набора чисел $a^{1}, \ldots, a^{m}$, то для доказательства связности $\mathbb{C} \backslash \Gamma$ достаточно рассмотреть чисто периодический случай, когда $a_{n m+l}=a^{l}$ при всех $n=0,1, \ldots, l=1, \ldots, m$. При этом можно предполагать, что $a^{l} \neq 0, l=1, \ldots, m$, так как в противном случае множество $\Gamma$ конечно, и $\mathbb{C} \backslash \Gamma$, очевидно, связно. Как уже отмечалось в $\S 1$, в чисто периодическом случае предельная функция $f(z)$ легко находится в явном виде как притягивающая неподвижная точка дробно-линейного преобразования $\widetilde{S}^{1}(w, z)=S^{1} \circ \cdots \circ S^{m}(w, z)$, где $S^{l}(w, z)=\frac{a^{l} z}{w+1}, l=1, \ldots, m$. В обозначениях теоремы 1 точки ветвления функции $f\left(z^{-1}\right)$ совпадают с точками ветвления функции

$$
\sqrt{I^{2}\left(z^{-1} ; a^{1}, \ldots, a^{m}\right)-4 a^{1} \ldots a^{m} z^{-m}} .
$$


По уже доказанному утверждению 6$)$ компакт $\Gamma^{-1}\left(a^{1}, \ldots, a^{m}\right)$ соединяет между собой пары точек ветвления $f\left(z^{-1}\right)$ таким образом, чтобы иметь наименьший трансфинитный диаметр среди всех таких соединений. Из этого экстремального свойства компакта $\Gamma^{-1}\left(a^{1}, \ldots, a^{m}\right)$ следует связность множества $\mathbb{C} \backslash \Gamma^{-1}\left(a^{1}, \ldots\right.$ $\left.\ldots, a^{m}\right)$, а следовательно, и множества $\mathbb{C} \backslash \Gamma\left(a^{1}, \ldots, a^{m}\right)$. Теорема 2 полностью доказана.

В заключение заметим, что теорема, аналогичная теореме 2 о сходимости правильных $C$-дробей с предельно периодическими коэффициентами, имеет место и для присоединенных непрерывных дробей с предельно периодическими коэффициентами. Действительно, множество сходимости таких дробей легко находится при помоши теоремы 1 , а экстремальное свойство множества особенностей таких дробей доказывается, как и вьше, при помоши теоремы Полиа и формул, выражающих коэффициенты присоединенной дроби через ганкелевы определители предельной функции (см., например, [6, с. 239]).

\section{Список литературы}

1. Vlek E. V. On the conergence of algebraic continued fractions whose coefficients have limiting values // Trans. Amer. Math. Soc. 1904. V. 5. P. 253-262.

2. Голузин Г. М. Геометрическая теория функций комплексного переменного. М.: Наука, 1966.

3. Stahl $\mathrm{H}$. Orthogonal polynomials with complex valued weight function. I.II // Constr. approx. 1986. V. 2. P. 225-240; P. 241-251.

4. Буслаев В.И. О теореме Пуанкаре и ее приложениях к вопросам сходимости цепных дробей // Матем. сб. 1998. Т. 189. № 12. С. 13-28.

5. Worpitsky J. Untersuchungen über die Entwickelung der monodromen und monogenen Funktionen durch Kettenbruche // Friedrichs-Gymnasium und Realschule Jahresbericht. Berlin, 1865. S. 3-39.

6. Джоунс У., Трон В. Непрерывные дроби. М.: Мир, 1985.

7. Polya G. Beitrag zur Verallgemeinerung des Verzerrungssatzes auf mehrfach zusammenhängende Gebiete. III // Sitzungsber. Preuss. Akad. Wiss. Phys.-math.Kl. 1929. S. 55-62.

Математический институт им. В. А. Стеклова РАН

Поступило в редакцию

7.XII. 2000 